\title{
An Efficient Approach for Robustness-based Design Optimization under Interval Uncertainty
}

\author{
Prithbey Raj Dey and Kais Zaman
}

\begin{abstract}
This paper proposes a methodology for robustnessbased design optimization under both aleatory (i.e., natural or physical variability) and epistemic uncertainty (i.e., imprecise probabilistic information). The proposed formulations specifically deal with epistemic uncertainty arising from multiple interval data. An efficient likelihood-based approach is used to represent the interval uncertainty, which is then used in the framework for robustness-based design optimization to achieve computational efficiency. The proposed robust design optimization methodology is illustrated using a general mathematical example problem.
\end{abstract}

Keywords - Likelihood-based estimation, robust design, interval data, epistemic uncertainty.

\section{Introduction}

In engineering applications, it is required to quantify the uncertainty in the input random variables. The system or process is generally expressed with the response or output function $f(\boldsymbol{x})$. The input random variables $\boldsymbol{x}$ of a response or output function $f(\boldsymbol{x})$ may contain both aleatory and epistemic uncertainty.

In the system or process design, there exist several methods, based on the aleatory uncertainty, including Monte Carlo simulation, first order reliability method (FORM), second order reliability method (SORM) etc. Epistemic uncertainty is produced due to lack of information about the system or process and due to lack of skill or knowledge for extracting data from a system or process. However, considering different situations, the epistemic uncertainty can be divided into two types: insufficient data with random characteristic [1] and insufficient data with deterministic characteristic [2]. Epistemic uncertainty can be reduced by gathering more information about the system which will tend to increase the cost. Therefore, this is important to estimate the uncertainty precisely in a cost effective way. This paper specifically focuses on epistemic uncertainty arising from multiple interval data on input random variables.

Prithbey Raj Dey

Dhaka University of Engineering and Technology, Bangladesh

Kais Zaman

Bangladesh University of Engineering and Technology, Bangladesh
There exist well-established methods for representation of aleatory uncertainty. However, in the complex engineering designs or processes, it is always difficult to obtain precise point data from the observations. There exists high probability of getting deviation in the manufactured data which will produce an interval or range of the experimental data. Therefore, experts and researchers have developed different methods to estimate the response of a system function when information on input variables are available in the form of interval data (e.g., [3-5]).

There are various sources of interval data which are mentioned in the literatures $[4,5]$. Interval data can be classified into single and multiple interval data considering computational method. In the multiple interval data, each interval generally bears equal weight [4]. Considering this fact, Empirical $p$-box can be formulated [7]. The Equiprobability model is popular for the representation of the interval data. This model corresponds to Laplacian principle of indifference [8], where each interval is treated using uniform distribution [9].

Likelihood-based methodology has been popular for the probabilistic representation of a random quantity where sparse point data and interval data are available $[10,11]$. Likelihoodbased methodology can quantify the distribution uncertainty for specific distributions (e.g. normal, lognormal, etc.) while fitting probability distributions to sparse and imprecise data [12]. Maximum likelihood parameter estimation is also used in several engineering applications [13-15]. Maximum likelihood method is proven to be more efficient than other methods in the application with different types of distribution. Type 1 Extreme Value Distribution of Maxima is used to design engineering systems [16].

The current research is intended to develop a methodology that provides decision support to engineers for design and analysis of engineering systems for poor amount of data considering stochastic epistemic uncertainty. This uncertainty prevails in the natural characteristics of a design and observed data and this is ignored in the deterministic design optimization. Non-deterministic design optimization has gained increasing attention in the last few decades due to this reason. There are now extensive volume of methods and applications available for non-deterministic design optimization problems. Robustness-based design [17-21] is the prominent field of optimization which considers the uncertainty in the design parameters. Robustness is the performance criteria for a system to operate continuously over 
Proc. of the Third Intl. Conf. on Advances in Mechanical, Aeronautical and Production Techniques - MAPT 2015

Copyright ( $\odot$ Institute of Research Engineers and Doctors, USA .All rights reserved.

ISBN: 978-1-63248-059-0 doi: 10.15224/ 978-1-63248-059-0-64

a wide range of operational conditions and will be failed outside the conditions [22].Taguchi developed the concept of robust design and proposed a method where the product performance or the output remains insensitive to the variation in design variables in manufacturing process [23]. The variation in the design variables was designated as noise which could be created from various factors in the manufacturing process. As engineering models are becoming more and more complex day by day, application of statistical design tools in Taguchi's method is not well enough to calculate optimal feasible solution for multiple measurements of performance and design constraints [24]. Due to application of nonlinear programming in the robust design, it became possible to achieve robustness in both performance outputs and design constraints [18].

There exist a few methods that develop robustness-based design optimization methodology under data uncertainty (i.e., using sparse point data and interval data on input random variables). Zaman et al. [20] proposed a decoupled approach for robustness-based design optimization using both sparse point and interval data. They achieved computational efficiency by un-nesting the design optimization from the uncertainty analysis of the epistemic variables. This is a sequential approach, where two optimization formulations are solved iteratively until convergence. In this paper, an optimization approach is proposed where the epistemic analysis is completely eliminated from the design optimization framework by estimating the distribution parameters of the epistemic variables using a likelihood-based uncertainty representation of interval data [25].

The rest of this paper is organized as follows. In section 2, a nested optimization-based methodology is presented that estimates the distribution parameters of input random variables under interval uncertainty using maximum likelihood-based approach. Section 3 illustrates the formulation of the robustness-based design optimization model for the MLE-based approach. Section 4 illustrates numerical example for the proposed MLE-based robust design optimization. Section 5 provides conclusions and suggestions for future work.

\section{Likelihood-based approach to epistemic uncertainty representation}

Likelihood-based approach has versatile applications, however, most of them are developed for point data. In this section, a maximum likelihood-based approach is discussed that estimates the uncertainty in input variable $x$ described by multiple interval data. Maximum likelihood estimation (MLE) constructs an estimator to estimate the unknown distribution parameters $(P)$. The 'likelihood' term was first introduced by an English mathematical statistician named R. A. Fisher in 1921. The likelihood for a parameter ' $P$ ' is a quantity proportional to the probability of the parameter $P$ quantified for a specific population where variable $x$ consists the observed data as a sample [26].

A Likelihood-based methodology has been developed to estimate the epistemic uncertainty from the interval data for any distribution in [25]. In this paper, we use this likelihood approach in robustness-based design optimization framework.

The likelihood-based approach for representation of epistemic uncertainty developed in [25] uses the following nested optimization formulation:

$$
\begin{aligned}
& \min _{x}\left(\max _{p}(f(x \mid \mathbf{P})=\log (L(x \mid \mathbf{P})))\right) \\
& \text { s.t. } \quad l b_{i} \leq x_{i} \leq u b_{i} \quad \text { for } i=1,2, \ldots, n
\end{aligned}
$$

We can estimate the parameters of the distribution of a random variable described by interval data by solving the optimization problem in Eq. (1). In the numerical example, we have used normal distribution for the sake of illustration.

Zaman et al. [5] formulated a method using nonlinear programming for calculating the moments from the different combination of multiple interval data. The bounds of the moments help to verify the parameters estimated from the maximum likelihood estimation method through producing bounds on parameters.

\section{Robustness-based design optimization under epistemic uncertainty}

The variables in the designs and processes are considered as fixed in the deterministic optimization formulation without considering any stochastic characteristics or data uncertainty in the variables. This deterministic design optimization could be used to get an optimal point which might be applied as an initial guess in the robustness-based optimization problem.

In real engineering applications, the robust design optimization must consider the stochastic characteristics of the data which may make it complex in nature. In the robustnessbased design optimization, objective robustness is achieved by measuring the variation in the objective function through the variance or standard deviation. Feasibility robustness is achieved by feasible region reduction method. First order Taylor series expansion is used to estimate the mean and variance of the objective function. Weighted sum method is used to trade off the multiple objectives in the performance function of the robust design optimization [20].

The robustness-based design optimization was initially designed only considering aleatory uncertainty. However, the design methodology includes complexity in the robust design 
Proc. of the Third Intl. Conf. on Advances in Mechanical, Aeronautical and Production Techniques - MAPT 2015

Copyright ( $\odot$ Institute of Research Engineers and Doctors, USA .All rights reserved.

ISBN: 978-1-63248-059-0 doi: 10.15224/ 978-1-63248-059-0-64

due to epistemic uncertainty. Therefore, the design variables $d$ and the input random variables $z$ might have a high chance for having epistemic uncertainty in the form of multiple interval data. Normally, the designer has no control on the non-design epistemic variables $z$. Hence, the optimization method has to employ a search among the possible values of the epistemic variables in order to find an optimal solution in the nested formulation of robustness-based design optimization. This nested formulation is a very expensive formulation and does not provide any guarantee for convergence.

Therefore, another design optimization algorithm is developed which is un-nested from the epistemic analysis with the computational accuracy. The decoupled approach [20] can achieve computational efficiency by un-nesting the design analysis from the epistemic analysis. We can achieve further computational efficiency if the uncertainty analysis for the epistemic variable is carried out outside the design optimization framework. In the following subsection, we have proposed an efficient approach for robustness-based design optimization, where epistemic analysis is done based on the likelihood-based approach described in section 2 .

The proposed formulation of nonlinear MLE-based robust design optimization can be expressed as:

$$
\begin{array}{ll}
d^{*}= & \arg \min \left(w \times \mu_{f}\left(d, \mu_{z}^{*}, \sigma_{z}^{*}\right)+v \times \sigma_{f}\left(d, \mu_{z}^{*}, \sigma_{z}^{*}\right)\right) \\
\text { s.t. } & L B+k \sigma\left(g_{i}\left(d, \mu_{z}^{*}, \sigma_{z}^{*}\right)\right) \leq E\left(g_{i}(d, z)\right) \leq U B-k \sigma\left(g_{i}\left(d, \mu_{z}^{*}, \sigma_{z}^{*}\right)\right) \\
& l b_{i}+k \sigma\left(x_{i}\right) \leq d_{i} \leq u b_{i}-k \sigma\left(x_{i}\right) \text { for all } i
\end{array}
$$

In the Eqn. (2), design variables are denoted by $d$ which is a vector and $z$ is the vector of the non-design epistemic variables. $E\left(g_{i}(d, z)\right)$ is the mean and $\sigma\left(g_{i}\left(d, \mu_{z}^{*}, \sigma_{z}^{*}\right)\right)$ is the standard deviation of the $i$ 'th constraints. $L B$ and $U B$ are the lower and upper bounds of the constraints $g_{i}$ 's, respectively where $l b$ and $u b$ are the vectors of the lower and upper bounds of the design variables. Here, the values of ' $w \geq 0$ ' and ' $v \geq 0$ ' are the weighting coefficients which represent the relative importance of the objectives $\mu_{f}$ and $\sigma_{f}$ where $k$ ensures the feasibility robustness in the constraints. The standard deviation $\left(\sigma_{d}\right)$ or variance of the design variables, the mean $\left(\mu_{z}\right)$ and standard deviation $\left(\sigma_{z}\right)$ of the non-design epistemic variables are provided as fixed values for robust design optimization. The estimated uncertainty of the non-design variables can be provided through the likelihood-based method.

The uncertainty representation method is shown with the illustration of normal distribution in section 2. The MLEbased approach estimated the parameters which can be used to provide the mean $\left(\mu_{z}\right)$ and standard deviation $\left(\sigma_{z}\right)$ of the nondesign epistemic variables.
The mean $(\mu)$ and standard deviation $(\sigma)$ of the performance function in Eqn. (2) can be obtained by first order Taylor series expansion method. If there is a response variable $Y$ which is represented by a non-linear performance function $f$ consists a set of random variables $\left(x_{1}, x_{2}, \ldots, x_{n}\right)$ then the response variable can be represented as,

$Y=f\left(x_{1}, x_{2}, \ldots, x_{n}\right)$

The first-order approximate mean of $Y$ :

$E(Y)=f\left(\mu_{x_{1}}, \mu_{x_{2}}, \ldots \ldots, \mu_{x_{n}}\right)$

The first-order variance of $Y$ can be written as:

$\operatorname{Var}(Y) \approx \sum_{i=1}^{n}\left(\frac{\partial f}{\partial x_{i}}\right)^{2} \operatorname{Var}\left(x_{i}\right)+\sum_{i=1}^{n} \sum_{\substack{j=1 \\ i \neq j}}^{n} \frac{\partial f}{\partial x_{i}} \frac{\partial f}{\partial x_{j}} \operatorname{Cov}\left(x_{i}, x_{j}\right)$

Using Eqns. (4) and (5), based on approximation method, the moments of the performance function can be estimated. These procedures are followed to estimate the mean and the standard deviation of the performance function in the robustness-based design optimization.

\section{Iv. Numerical example}

In this paper, maximum likelihood-based estimation (MLE) approach has been proposed for robustness-based design optimization. The MLE-based robust design optimization is illustrated with normal distribution. A mathematical design problem is illustrated which includes an objective function of three random variables and four coefficients. The observed data of the coefficients are multiple intervals which are overlapping or non-overlapping in nature.

The example problem has two nonlinear constraints. There are three design variables $\boldsymbol{x}$ and four coefficients $(a, b, c$ and $l)$ in the performance function. The variables $x_{i}$ (where, $i=1,2,3$ ) are treated as design variables where the coefficients are treated as the non-design epistemic variables and denoted as $z_{i}$.

The performance function with the nonlinear constraints of the mathematical model:

$$
\begin{array}{ll}
\min & f(x)=a x_{1}{ }^{2}-b x_{1} x_{2}+c x_{1} x_{2}{ }^{2}+l x_{1} x_{3} \\
\text { s.t. } \quad & -2 x_{1}{ }^{2}-x_{1} x_{2}+x_{3} \leq-1 \\
& -x_{1} x_{2}+x_{2}{ }^{2}-x_{1} x_{3} \leq-3
\end{array}
$$

The coefficients of the objective function consist of multiple interval data. The parameters of the log likelihood density function are estimated. The interval data including 
Proc. of the Third Intl. Conf. on Advances in Mechanical, Aeronautical and Production Techniques - MAPT 2015

Copyright ( $\odot$ Institute of Research Engineers and Doctors, USA .All rights reserved.

ISBN: 978-1-63248-059-0 doi: 10.15224/ 978-1-63248-059-0-64

overlapping, non-overlapping and mixed of the coefficients are presented in Table 1.

TABLE I. Multiple interval data of the coefficients

\begin{tabular}{|c|c|}
\hline Coefficient & Multiple Interval Data $\left[l b_{i}-u b_{i}\right]$ \\
\hline$a$ & {$[1.5-2.2 ; 1.8-2.3 ; 2.0-2.5 ; 2.1-2.6 ; 2.2-2.7]$} \\
\hline$b$ & {$[2.8-3.2 ; 3.3-3.9 ; 4.0-4.5 ; 4.7-5.0 ; 5.2-6.0]$} \\
\hline$c$ & {$[2.7-3.1 ; 2.9-3.2 ; 2.8-3.2 ; 3.5-3.8 ; 4.0-4.5]$} \\
\hline$l$ & {$[4.8-5.1 ; 5.3-5.9 ; 6.0-6.8 ; 6.5-6.9 ; 6.6-7.0]$} \\
\hline
\end{tabular}

The parameters of the coefficients $(a, b, c$ and $l)$ are estimated from Eqn. (1) through nested optimization which are given in Table 2.

TABLE II. Parameters of normal distribution for the coefficients

\begin{tabular}{|c|c|c|}
\hline Coefficient & $\mu_{z}$ & $\sigma_{z}$ \\
\hline$a$ & 2.2200 & 0.4792 \\
\hline$b$ & 4.2200 & 1.1565 \\
\hline$c$ & 3.3400 & 0.7003 \\
\hline$l$ & 6.1600 & 0.9222 \\
\hline
\end{tabular}

In the proposed robust design optimization, it requires the standard deviations $\left(\sigma_{x}\right)$ of the design variables and the moments (mean $\left(\mu_{z}\right)$ and standard deviation $\left(\sigma_{z}\right)$ ) of the epistemic non-design variable.

We have assumed the standard deviation of the random variables as,

$$
\sigma_{x_{1}}=\sigma_{x_{2}}=\sigma_{x_{3}}=0.5
$$

The robust design formulation from Eqn. (2) for the mathematical example:

$$
\begin{array}{ll}
d^{*}= & \underset{d}{\arg \min }\left(w \times \mu_{f}\left(d, \mu_{z}^{*}, \sigma_{z}^{*}\right)+(1-w) \times \sigma_{f}\left(d, \mu_{z}^{*}, \sigma_{z}^{*}\right)\right) \\
\text { s.t. } & L B+k \sigma\left(g_{i}\left(d, \mu_{z}^{*}, \sigma_{z}^{*}\right)\right) \leq E\left(g_{i}(d, z)\right) \leq U B-k \sigma\left(g_{i}\left(d, \mu_{z}^{*}, \sigma_{z}^{*}\right)\right) \\
& l b_{i}+k \sigma\left(x_{i}\right) \leq d_{i} \leq u b_{i}-k \sigma\left(x_{i}\right) \quad \text { for } i=1,2,3
\end{array}
$$

The mean $\left(\mu_{f}\right)$ and standard deviation $\left(\sigma_{f}\right)$ of the performance function are estimated form first order Taylor series expansion method. As mentioned previously, ' $w \geq 0$ ' is the weighting coefficient which ranges from $O$ to $l$ that provides the relative importance of the objectives $\mu_{f}$ and $\sigma_{f}$. The value of ' $k$ ' is assumed to be unity which adjusts the robustness of the method against any types of conservatism in the solution. The value of ' $k$ ' ensures feasibility robustness in the robust design optimization.

Matlab solver 'fmincon' is used for solving robust design optimization in Eqn. (7). Here, 'fmincon' uses 'Active set algorithm' for optimization of the performance function. From the optimization, mean $\left(\mu_{f}\right)$ and standard deviation $\left(\sigma_{f}\right)$ of the performance function are obtained for different values of ' $w$ '. In order to demonstrate the accuracy and efficiency of the proposed methodology, this example problem is also solved using the decoupled method developed in Zaman et al. [20]. Results obtained from both the approaches are presented in Figure 1.

It is seen in Figure 1 that MLE-based robust design generates superior results for this example problem as evident from the lower values of both mean and standard deviation of the performance function. Since the decoupled approach developed in Zaman et al. [20] solved two optimization problems (one for robust design under aleatory uncertainty and the other for robust design for non-design epistemic variables) iteratively until convergence, the computational expense for the proposed MLE-based robust design would be approximately one-fourth of the former assuming that it requires at least two iterations between the two optimization formulations for convergence.

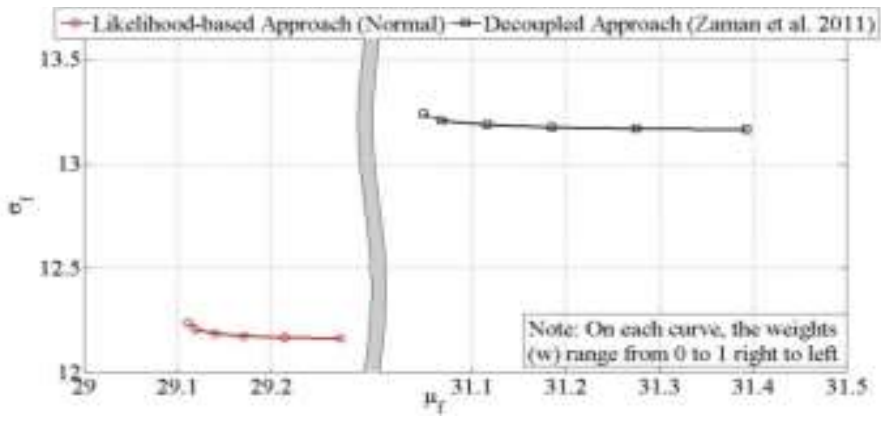

Figure 1. Robust design optimization using MLE-based and decoupled approaches

\section{v.Summary and Conclusion}

This paper proposed a likelihood-based robust design optimization under epistemic uncertainty arising from multiple interval data. The maximum likelihood-based uncertainty representation methodology is used in the design optimization framework to solve robustness-based design optimization problem under epistemic uncertainty. This proposed methodology is illustrated for a numerical example.

The major contribution of this paper is to develop a single loop robustness-based design optimization framework using the likelihood-based uncertainty representation for the nondesign epistemic variables. The likelihood-based approach of epistemic uncertainty representation is able to estimate the 
Proc. of the Third Intl. Conf. on Advances in Mechanical, Aeronautical and Production Techniques - MAPT 2015

Copyright (C) Institute of Research Engineers and Doctors, USA .All rights reserved.

ISBN: 978-1-63248-059-0 doi: 10.15224/ 978-1-63248-059-0-64

parameters of any distributions. The estimated parameters can be used to calculate moments of the distributions which are required for the robust design optimization.

The proposed MLE-based robust design optimization model is better than the existing robust optimization methods including decoupled approach with respect to accuracy and computational expense. The proposed MLE-based robust optimization can provide most probable solution of the robust design where decoupled approach provides the worst case scenario due to the use of upper bound of the moment (standard deviation). The proposed robust optimization methodology reduces the computational cost through a single loop formulation.

This paper developed a robust design optimization methodology for single discipline system. However, the proposed methodology can easily be extended to solve robust design optimization problems for multidisciplinary systems.

\section{References}

[1] C. Baudrit and D. Dubois, (2006) "Practical representations of incomplete probabilistic knowledge", Computational Statistics \& Data Analysis, Vol 51, pp. 86-108.

[2] J. C. Helton, J. D. Johnson and W. L. Oberkampf, (2004) "An exploration of alternative approaches to the representation of uncertainty in model predictions", Reliability Engineering and System Safety, Vol 85, pp 39-71.

[3] K. Zaman, M. McDonald and S. Mahadevan, (2011) "Probabilistic Framework for Uncertainty Propagation with both Probabilistic and Interval Variables", ASME Journal of Mechanical Design, 133(2):0210101-02101014.

[4] S. Ferson, V. Kreinovich, J. Hajagos, W. Oberkampf and L. Ginzburg, (2007) "Experimental uncertainty estimation and statistics for data having interval uncertainty", Sandia National Laboratories Technical report SAND2007-0939, Albuquerque, NM.

[5] K. Zaman, S. Rangavajhala, M. McDonald and S. Mahadevan, (2011) "A probabilistic approach for representation of interval uncertainty”, Reliability Engineering \& System Safety”, vol. 96, no. 1, pp. 117-130.

[6] X. Du, A. Sudjianto and B. Huang, (2005) "Reliability based design with mixture of random and interval variables", Journal of Mechanical Design, Vol 127, pp. 1068-76.

[7] R. C. Williamson and T. Downs (1990) "Probabilistic arithmetic I: numerical methods for calculating convolutions and dependency bounds", International Journal of Approximate Reasoning 1990, Vol 4, pp. $89-158$.

[8] C. Howson and P. Urbach, (1993) Scientific reasoning: the Bayesian approach. 2nd ed. Chicago, IL: Open Court.

[9] P. Bertrand and F. Groupil, (2000) Descriptive statistics for symbolic data, In: Bock HH, Diday E, editors. Analysis of symbolic data. Berlin: Springer.

[10] S. Sankararaman and S. Mahadevan, (2011) "Likelihood-based representation of epistemic uncertainty due to sparse point data and/or interval data", Reliability Engineering and System Safety, Vol 96, pp. 814-824.

[11] S. Sankararaman and S. Mahadevan, (2012) "Likelihood-Based Approach to Multidisciplinary Analysis Under Uncertainty”, Reliability Engineering \& System Safety, Volume 134, Issue 3.

[12] S. Sankararaman and S. Mahadevan (2013) "Distribution type uncertainty due to sparse and imprecise data", Mechanical Systems and Signal Processing, Volume 37, Issues 1-2, May-June 2013, pp. 182198.

[13] J. F. Monaco, D. S. Kidman, D. J. Malloy, D. G. Ward and J. F. Gist IV, (2008) "Automated Methods to Calibrate a High-Fidelity Thrust
Deck to Aid Aeropropulsion Test and Evaluation", ASME Turbo Expo 2008: Power for Land, Sea, and Air, Paper No. GT2008-50213, pp. 4154; 14 pages, doi:10.1115/GT2008-50213.

[14] J. P. Modisette (2012) "Maximum Likelihood Approach to State Estimation in Online Pipeline Models", 9th International Pipeline Conference, Paper No. IPC2012-90664, pp. 813-824; 12 pages, doi:10.1115/IPC2012-90664.

[15] J. Xiao and B. Kulakowski, (2003) "Hybrid Genetic Algorithm: A Robust Parameter Estimation Technique and Its Application to Heavy Duty Vehicles", ASME 2003 International Mechanical Engineering Congress and Exposition, Paper No. IMECE:2003-41934, pp. 10871096; 10 pages, doi:10.1115/IMECE2003-41934.

[16] P. H. Wirsching and L. H. Jones (1976) "On The Use of The Extreme Value Distribution in Reliability Analysis and Design", J. Manuf. Sci. Eng. 98(3), 1080-1085 (Aug 01, 1976) (6 pages)doi:10.1115/1.3439011.

[17] A. Parkinson, C. Sorensen and N. Pourhassan (1993) "A general approach for robust optimal design", Trans ASME 115:74-80.

[18] X. Du and W. Chen (2000) "Towards a better understanding of modeling feasibility robustness in engineering", ASME J Meach Des 122(4):385-394.

[19] I. Doltsinis and Z. Kang (2004) "Robust design of structures using optimization methods", Comput Methods ApplMechEng, vol 193, pp. $2221-2237$.

[20] K. Zaman, M. McDonald, S. Mahadevan and L. Green, (2011) "Robustness-based design optimization under data uncertainty. Structural and Multidisciplinary Optimization", vol. 44, no. 2, pp. 183-197.

[21] K. Zaman and S. Mahadevan (2013) "Robustness-based Design Optimization of Multidisciplinary System under Epistemic Uncertainty", AIAA Journal, Volume 51, Issue 5, pp. 1021-1031.

[22] S. D. Gribble (2001) "Robustness in Complex Systems", Proceedings of the 8th Workshop on Hot Topics in Operating Systems (HotOS-VIII).

[23] G. Taguchi (1993) Taguchi on robust technology development: bringing quality engineering upstream. ASME, New York.

[24] D. L. Wei, Z. S. Cui and J. Chen (2009) "Robust optimization based on a polynomial expansion of chaos constructed with integration pointn rules", J MechEngSci 223(5):1263-1282 (Part C).

[25] P. R. Dey and K. Zaman (2014) "Likelihood-based Approach to Representation of Interval Uncertainty", $15^{\text {th }}$ National Statistical Conference, Bangladesh Statistical Association, December 25-26, Dhaka, Bangladesh.

[26] R. A. Fisher, (1921) "On the probable error of a coefficient of correlation deduced from a small sample”, Metron, Vol 1, pp. 3-32.

About Authors:

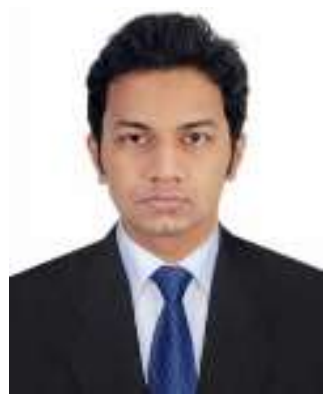

Prithbey Raj Dey is a Lecturer at Dept. of Industrial \& Production Engineering, Dhaka University of Engineering and Technology, Bangladesh. He received his B. Sc. And M. Sc. degrees in Industrial \& Production Engineering from Bangladesh University of Engineering and Technology in 2012 and 2015, respectively. His research interests include robust and reliability-based design optimization, and operations research.

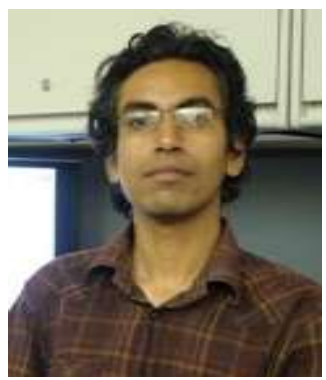

Dr. Kais Zaman is an Associate Professor at Bangladesh University of Engineering and Technology, Dhaka, Bangladesh. He received his Ph.D. from Vanderbilt University, Nashville, TN, USA. His research interests include multidisciplinary optimization, risk and reliability analysis, operations research, and financial engineering. 